\title{
An Extract of Chinpi, the Dried Peel of the Citrus Fruit Unshiu, Enhances Axonal Remyelination via Promoting the Proliferation of Oligodendrocyte Progenitor Cells
}

\author{
Hideaki Tokunaga, ${ }^{1}$ Chika Seiwa, ${ }^{1}$ Nozomu Yoshioka, ${ }^{2}$ Kazushige Mizoguchi, ${ }^{3}$ \\ Masahiro Yamamoto, ${ }^{1,3}$ Hiroaki Asou, ${ }^{1}$ and Sadakazu Aiso ${ }^{4}$ \\ ${ }^{1}$ Center for Kampo Medicine, School of Medicine, Keio University, 35 Shinanomachi, Shinjuku-ku, Tokyo 160-8582, Japan \\ ${ }^{2}$ Department of Brain Development and Neural Regeneration, Tokyo Metropolitan Institute of Medical Science, 2-1-6 Kamikitazawa, \\ Setagaya-ku, Tokyo 156-8506, Japan \\ ${ }^{3}$ Tsumura Research Laboratories, Tsumura \& Co., 3586 Yoshiwara, Amimachi, Inashiki, Ibaraki 300-1192, Japan \\ ${ }^{4}$ Department of Anatomy, School of Medicine, Keio University, 35 Shinanomachi, Shinjuku-ku, Tokyo 160-8582, Japan
}

Correspondence should be addressed to Masahiro Yamamoto; hirokoma@h.email.ne.jp

Received 25 November 2015; Revised 31 January 2016; Accepted 2 February 2016

Academic Editor: Karen Nieber

Copyright (C) 2016 Hideaki Tokunaga et al. This is an open access article distributed under the Creative Commons Attribution License, which permits unrestricted use, distribution, and reproduction in any medium, provided the original work is properly cited.

\begin{abstract}
The aging-induced decrease in axonal myelination/remyelination is due to impaired recruitment and differentiation of oligodendrocyte progenitor cells (OPCs). Our previous studies have shown that a monoclonal antibody to DEAD (Asp-Glu-AlaAsp) box polypeptide 54 (Ddx54), a member of the DEAD box family of RNA helicases, (1) specifically labels oligodendrocyte lineages, (2) binds to mRNA and protein isoforms of myelin basic proteins (MBP), and (3) regulates migration of OPCs from ventricular zone to corpus callosum in mice. It has also been demonstrated that specific loss of a $21.5 \mathrm{kDa} \mathrm{MBP}$ isoform (MBP21.5) reflects demyelination status, and oral administration of an extract of Chinpi, citrus unshiu peel, reversed the aging-induced demyelination. Here, we report that Chinpi treatment induced a specific increase in the MBP21.5, led to the reappearance of Ddx54expressing cells in ventricular-subventricular zone and corpus callosum of aged mice, and promoted remyelination. Treatment of in vitro OPC cultures with Chinpi constituents, hesperidin plus narirutin, led to an increase in 5-bromo-2'-deoxyuridine incorporation in Ddx54-expressing OPCs, but not in NG2- or Olig2-expressing cell populations. The present study suggests that Ddx54 plays crucial role in remyelination. Furthermore, Chinpi and Chinpi-containing herbal medicines may be a therapeutic option for the aging-induced demyelination diseases.
\end{abstract}

\section{Introduction}

Myelin is the coiled cell membrane that insulates the axons of nerve fibers. In the central nervous system (CNS), myelin is synthesized by oligodendrocytes, and in rodents, the majority is produced during the first six postnatal weeks after proliferation of oligodendrocyte progenitor cells (OPCs). OPCs persist in the brain parenchyma of the adult mouse CNS, comprising approximately $\sim 5 \%$ of all neural cells [1]. Regeneration of the myelin sheath (remyelination) occurs as a spontaneous response to neuronal demyelination. Parenchymal OPCs continue to divide and differentiate into myelinating oligodendrocytes throughout life [2], playing a crucial role in the repair process [3]. Neural stem cells (NSCs) within the ventricular-subventricular zone [4-7] also maintain their ability to generate oligodendrocytes and promote remyelination $[8,9]$. However, similar to other body repair processes, remyelination becomes less efficient with age [10-12].

There is a general consensus that CNS remyelination involves the recruitment and differentiation of OPCs, which facilitates the development of new oligodendrocytes $[6,13$, 14], rather than to new processes formation by previously myelinating oligodendrocytes $[3,15]$. In response to demyelination, OPCs are recruited to the lesion site, followed by proliferation, migration, and rapid differentiation in the demyelinated area. A key factor that causes insufficient remyelination, 
in various demyelinating diseases as well as aging-induced demyelination, is deficient OPC proliferation $[10,16]$. Several growth factors affect the in vitro proliferation, migration, and differentiation of OPCs [17], including platelet derived growth factor $\alpha$ and fibroblast growth factor-2, both of which promote the proliferation and motility of adult OPC $[18,19]$. However, the molecules and mechanisms that drive OPCs recruitment after demyelination remain largely unclarified.

In a previous study, we reported that a monoclonal antibody 4F2 recognizes Ddx54, a member of the DEADbox protein family that specifically stains oligodendrocyte lineages at the early embryonic stage through to adulthood. Ddx54 is expressed in neural tube cells at the earliest stage of oligodendrocyte lineage development (e.g., E 9.5 in rats), before they migrate away from the subventricular zone, suggesting that Ddx54 may be implicated [20]. Accordingly, a pilot study demonstrated that adenoviral vector-mediated knockdown of Ddx54 expression inhibited translocation of OPCs from the subventricular zone to the emerging white matter in mice [21]. Interestingly, Ddx54 protein interacted with both the mRNA and protein forms of myelin basic protein (MBP) [20, 21]. A $21.5-\mathrm{kDa}$ isoform of phosphorylated MBP, which was closely related to myelination status, was identified in lipid-rafts of myelin, while the absence of this isoform corresponded to cuprizone- and aging-induced demyelination [22, 23] as well as Ddx54 knockdown [21].

In another project investigating the potential efficacy of herbal medicines for demyelinating diseases, Chinpi, which is derived from the peel of citrus fruit unshiu [24], and Chinpi-containing Kampo (traditional Japanese) medicine [22] inhibited both cuprizone- and aging-induced demyelination via an $\mathrm{FcR} \gamma$-Fyn signaling cascade.

In this study, the in vivo effect of Chinpi on demyelination and Ddx54-expressing oligodendrocyte linages was investigated in the ventricular-subventricular zone of the lateral ventricles and the corpus callosum of aged mice and the in vitro effects were examined using OPC cultures.

\section{Materials and Methods}

2.1. Mice. Twenty-eight-month-old C57BL/6 mice were supplied by the department of Animal science, Tokyo Metropolitan Institute of Gerontology, and acclimated for one week in the animal experimental research laboratory and then randomly divided into two groups of five mice each. Mice were placed individually in plastic cages and subjected to a $12 \mathrm{hr}$ light $/ 12 \mathrm{hr}$ dark cycle at $24 \pm 1^{\circ} \mathrm{C}$ temperature and $55 \pm 5 \%$ relative humidity. Mice had access to food and water ad libitum. Chinpi was administered by dissolving the extract in drinking water at $0.5 \%$ weight/volume $[22,24]$. The mean amount of Chinpi consumed via drinking water was $4.1 \mathrm{~mL} \pm 0.2 \mathrm{~mL} /$ day/mouse. The mice received Chinpi for two months, from 28 to 30 months of age. All animal experiments were performed with the approval of the guidelines of the Institutional Animal Care and Use Committee of Keio University School of Medicine (approval number 08071).

2.2. Drugs and Reagents. Dried extracts made from dried peels, the citrus fruit unshiu, were prepared by Tsumura \&
Co. (Tokyo Japan). In brief, approximately $20 \mathrm{~g}$ of the dried peel was immersed in $200 \mathrm{~mL}$ of distilled water and heated to boiling point. After $1 \mathrm{~h}$ at $100^{\circ} \mathrm{C}$, followed by cooling to room temperature, the extracts were filtered and lyophilized to obtain the dried extract. The extracts dried extracts were subsequently examined for contents of flavonoids and used for in vitro and in vivo studies. Three-dimensional profiles of the chemical compounds detected within Chinpi extract were reported in a previous paper [24]. Hesperidin, narirutin (two major components of Chinpi extract), and other reagents were purchased from Wako Pure Chemical Industry, Ltd. (Osaka, Japan) unless otherwise stated.

2.3. Immunohistochemistry. All mice were deeply anesthetized with ether and perfused with $4 \%$ paraformaldehyde and stored in a fixative solution containing $0.2 \%$ glutaraldehyde in $0.2 \mathrm{M}$ phosphate buffer $(\mathrm{pH} 7.4)$. The cerebral hemispheres were separated and serial $10 \mu \mathrm{m}$ coronal sections were cut on a microtome. Immunohistochemistry was performed as previously described $[25,26]$. Free-floating sections were initially rinsed in $20 \mu \mathrm{M}$ phosphate-buffered saline (PBS) and incubated in a mixture of $3 \%$ hydrogen peroxide and $0.1 \%$ TritonX-100 for $15 \mathrm{~min}$ at room temperature. After rinsing in $20 \mathrm{mM}$ PBS, the sections were incubated overnight at $4^{\circ} \mathrm{C}$ with the primary antibody (4F2 or anti-MBP, see below) which was diluted with $20 \mathrm{mM}$ PBS-containing $0.5 \%$ skimmed milk. After rinsing in $20 \mathrm{mM}$ PBS for $15 \mathrm{~min}$, sections were incubated with biotinylated secondary antibody (1:100, Vector Laboratories) for $30 \mathrm{~min}$ at $37^{\circ} \mathrm{C}$. The sections were rinsed with $20 \mathrm{mM}$ PBS for $15 \mathrm{~min}$ and subsequently incubated in avidin-biotin peroxidase complex (Vectastain $\mathrm{ABC}$ kit, Vector Laboratories) for $30 \mathrm{~min}$ at $37^{\circ} \mathrm{C}$. After rinsing in $20 \mathrm{mM}$ PBS, immunoreactions were visualized in a solution containing $0.01 \%$ diaminobenzidine tetrahydrochloride and $0.01 \%$ hydrogen peroxide in $50 \mathrm{mM}$ Tris buffer $(\mathrm{pH}$ 7.4 ) at $37^{\circ} \mathrm{C}$ for 5 to $10 \mathrm{~min}$. The nuclei were counterstained with hematoxylin according to the manufacturer's instructions. Sections were mounted on MAS-coated glass slides (Matsunami Glass, Osaka, Japan), air-dried on a hot plate at $40^{\circ} \mathrm{C}$, and coverslipped with Entellan New (Merck, Darmstadt, Germany) after dehydration with ethanol and xylene. The primary antibodies, anti-Ddx54 mouse monoclonal antibody 4F2 [21] $(1: 200)$ and anti-MBP rabbit polyclonal antibody [27], (1:500) were prepared in our laboratory.

2.4. Toluidine Blue Staining. Mice were perfused with $4 \%$ paraformaldehyde and the brains were fixed overnight in an acid-alcohol solution (95\% ethanol/5\% acetic acid, v/v). The brain tissues were embedded in paraffin and sliced into $10 \mu \mathrm{m}$ thick sections. The sections were then mounted and stained for myelin using Luxol ${ }^{\circledR}$ Fast Blue (LFB: Acros Organics, Fair Lawn, NJ, USA) solution (0.1\% LFB in 95\% ethanol containing $0.05 \%$ acetic acid) and stained overnight at $60^{\circ} \mathrm{C}$. Toluidine blue staining was performed according to the manufacturer's protocol (Wako Pure Chemical Industry).

2.5. Electron Microscopy. Each cerebrum was fixed with $2.5 \%$ glutaraldehyde and then postfixed with $1 \% \mathrm{OsO}_{4}$. After dehydration in ethanol, the specimens were embedded 
in Quetol 812 (Nisshin EN, Tokyo, Japan) and ultrathin sections stained with $2 \%$ uranylacetate and lead solution were observed using a JOEL 100C electron microscope (JOEL, Tokyo, Japan), as described elsewhere. For the G-ratio measurement (the ratio of the axon diameter to the diameter of the axon plus the surrounding myelin), at least three mice per group were used. Between 8 and 10 microphotographs at a high magnification $(\times 5000$ or $\times 10000)$ of the coronal sections of the corpus callosum at the midline were taken for each mouse, and the $G$-ratios of at least 90 axons were measured. Axons with aberrant morphology, including myelin reduplication, abnormal splitting of the myelin sheath, vacuolization of the myelin lamellae, and myelin balloon formation, were excluded because these types of aberrant myelin morphology make it difficult to accurately evaluate the degree of myelination. For example, the extraordinarily large myelin sheath of axons with an abnormal morphology arising from the repetition of unaccomplished remyelination should not be estimated in the same manner as the myelin sheaths of normal axons. An additional quantitative study was undertaken to assess the number of myelinated fibers per $400 \mu \mathrm{m}^{2}$ using three mice per group.

2.6. Preparation of Purified OPCs. The method of Seiwa et al. was used for mouse OPC culture, as described [28]. After washing with Dulbecco's modified Eagle's medium (DMEM, Invitrogen Carlsbad, CA), cerebral hemispheres from E 17 mice were enzymatically dissociated in a mixture of $0.3 \%$ dispase II and $0.05 \%$ DNase (Roche Molecular Biochemicals, Mannheim, Germany) in DMEM. The dissociated cells were sieved through a $70 \mu \mathrm{m}$ pore nylon mesh (number 2350, Becton Dickson, Franklin, NJ) and then seeded on poly-L-lysine (PLL, ICN) coated $10 \mathrm{~cm}$ diameter culture dishes (Greiner Bio One, Tokyo, Japan) at a density of $1.3 \times$ $10^{7}$ cells/dish in DMEM containing 10\% FBS. After 5 days of culture, the cells were passaged with $0.2 \%$ trypsin in PBS and centrifuged for $10 \mathrm{~min}$ at $1,000 \mathrm{rpm}$ at $4^{\circ} \mathrm{C}$ in a $15 \mathrm{~mL}$ tube. The cells were resuspended in a serum-free medium ("BS" medium) consisting of DMEM supplemented with glucose $(5.6 \mathrm{mg} / \mathrm{mL})$, kanamycin $(60 \mathrm{mg} / \mathrm{mL})$, insulin $(5 \mu \mathrm{g} / \mathrm{mL})$, transferrin $(0.5 \mu \mathrm{g} / \mathrm{mL})$, BSA $(100 \mu \mathrm{g} / \mathrm{mL})$, progesterone $(0.06 \mathrm{ng} / \mathrm{mL})$, putrescine $(16 \mu \mathrm{g} / \mathrm{mL})$, sodium selenite $(40 \mathrm{ng} / \mathrm{mL})$, and thyroxine $(40 \mathrm{ng} / \mathrm{mL})$ and incubated for $2 \mathrm{hr}$ at $37^{\circ} \mathrm{C}$ in a $\mathrm{CO}_{2}$ incubator at a density of $2.5 \times 10^{6}$ cells $/ \mathrm{mL}$. These procedures are essential to eliminate neurons, microglia, and astrocytes. The cells were then thoroughly resuspended in serum-free medium and cultured at a density of $2 \times 10^{6} / \mathrm{mL}$ cells on a noncoated, $10 \mathrm{~cm}$ dish for 2 days. Immunocytochemistry demonstrated that more than $95 \%$ of the cultured cells were routinely determined to be positive for both OPC markers, Olig2 (1:200, goat polyclonal; R\&D Systems, Minneapolis, MN) and NG2 (1:200; rabbit polyclonal, a gift from Dr. William Stallcup, Bumham Institute for Medical Research, La Jolla, CA) by immunocytochemistry.

2.7. 5-Bromo- $2^{\prime}$-deoxyuridine (BrdU) Incorporation. The cells were pulsed with $20 \mu \mathrm{M}$ BrdU (Sigma Aldrich) for $48 \mathrm{hr}$, fixed with $70 \%$ ethanol for $30 \mathrm{~min}$ at $22^{\circ} \mathrm{C}$, rinsed with $0.05 \%$ BSA in PBS, and then treated with $2 \mathrm{~N} \mathrm{HCl}$ for $10 \mathrm{~min}$ at room temperature to denature the DNA in the preparation. The sample was then treated with $0.1 \mathrm{M}$ sodium tetraborate $(\mathrm{pH} \mathrm{8.5)}$ to neutralize $\mathrm{HCl}$. Cells were incubated with a mouse IgG antibody to BrdU (1:300; Dako, Glostrup, Denmark) for $2 \mathrm{~h}$ and incubated with the FITC-conjugated goat anti-mouse IgG secondary antibody $(1: 300)$ for $90 \mathrm{~min}$ at room temperature. Cell cycle analysis was performed using Cycletest Plus DNA reagent kit (Becton Dickson) according to the supplier's protocol.

2.8. Western Blot Analysis. Western blot analysis was performed as described previously [28]. Samples (brain homogenate, cell lysate) were separated using SDS-PAGE (4$20 \%$ gel gradient) for $2 \mathrm{~h}$ and then prepared for immunoblotting by semi-dry transfer onto an Immobilon PVDF membrane (Millipore, Bedford, MA, USA). The membranes were blocked with 5\% skimmed milk (Difco Laboratories, Detroit, MA, USA) in Tris-buffered saline ( $\mathrm{pH} \mathrm{7.6)}$ for $1 \mathrm{hr}$, and the blots were incubated with $4 \mathrm{~F} 2$, anti-MBP or anti- $\beta$ actin (Sigma Aldrich, St. Louis, MO) antibodies for $2 \mathrm{hr}$. Next the membranes were washed three times for $10 \mathrm{~min}$ each using $1 \%$ skimmed milk in Tris-buffered saline, incubated with alkaline phosphatase-conjugated goat anti-mouse or anti-rabbit IgG (diluted 1:800) for $2 \mathrm{~h}$, and developed using nitro blue tetrazolium and 15-bromo-4-chloro-3-indolylphosphate solution (both from Sigma, St Louis, MO, USA).

2.9. Statistics. Morphological data are represented as mean \pm SEM. Differences among groups were analyzed using Student's $t$-test. $P<0.05$ is considered statistically significant.

\section{Results}

3.1. Effect of Chinpi on Aging-Induced Demyelination. After the two-month administration period, the gray and white matter of the aged 30-month-old mice in the control and Chinpi groups were examined. Immunohistochemistry with the anti-MBP antibody stained the corpus callosum to the pia mater, but immunoreactivity was more intense in the Chinpitreated mice than the age-matched control mice (Figure 1). Observation of myelinated axons by toluidine blue staining (Figure 2(a)) and electron microscopy (Figure 2(b)) revealed the presence of aging-induced demyelination in the control mice, while remyelination was stimulated in Chinpi-treated mice. Quantitative analyses of the numbers of myelinated fibers (Figure 2(c)) and G-ratio (Figure 2(d)) demonstrated that both the number of myelinated axons and the thickness of the myelin sheath were increased in Chinpi-treated mice, presumably because of enhanced remyelination. Similar to previous reports using extremely old rodents ( 31 months), the brains of untreated aged mice showed many abnormalities in the myelin sheath, including myelin balloon formation, splitting of the myelin sheath, and vacuolization of myelin lamellae [29]. In contrast, a marked reduction in abnormal histological findings was observed in the brain of Chinpitreated mice. These results are consistent with our previous research using mice fed on a diet containing 1\% Chinpi for two months [24]. 


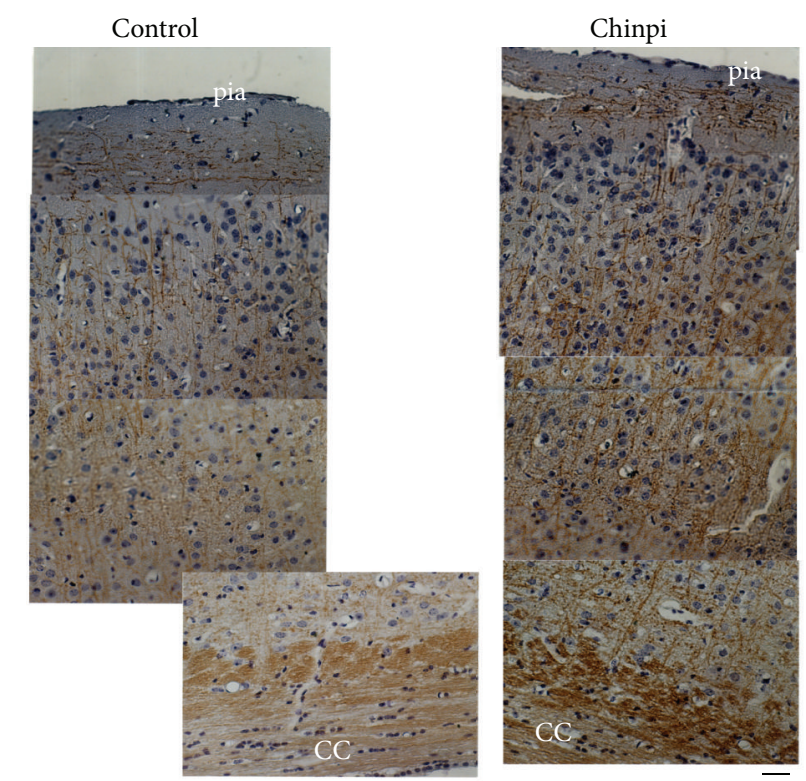

FIGURE 1: Immunohistochemistry of coronal section of the cerebrum using anti-MBP antibody. Twenty-eight-month-old mice were treated with or without Chinpi in drinking water for 2 months. An overview of the gray and white matter at 30 months old is shown. Myelin stained with anti-MBP antibody (brown signals) reveals the vertical, myelinated nerve fibers running from the corpus callosum (CC) to the pia matter. The number of myelinated nerve fibers was increased after two months of Chinpi, and age-induced demyelination was reduced compared with controls. Scale bar = $28 \mu \mathrm{m}$.

3.2. Effect of Chinpi on Ddx54-Expressing OPCs. The effect of Chinpi on Ddx54-expressing cells in the area of ventricular zone region facing the side of the lateral ventricles was investigated. Ddx54 immunoreactivity was scarcely detected in the control elderly (both 28- and 30-month old) mice as shown in Supplementary Fig. S1 in Supplementary Material available online at http://dx.doi.org/10.1155/2016/8692698 and Figure 3, respectively. Surprisingly, in Chinpi-treated mice, clear Ddx54 immunoreactivity was detected in the medial and lateral wall of the lateral ventricles at the most anterior and posterior regions (Figure 3). This pattern of Ddx54 labeling coincided with that of A2B5-positive cells in the same region of young to middle-aged mice $[30,31]$ as well as that of Ddx54-positive cells in younger mice [20], which represent OPC in this region. The appearance of Ddx54-expressing cells with the morphology of immature/mature oligodendrocytes was also observed in the corpus callosum (Figure 4). Accordingly, immunoblot analysis also indicated that the level of Ddx54 protein was higher in the brain homogenate of Chinpi-treated mice (Figure 5). The specific decrease in $21.5 \mathrm{kDa}$ isoform of MBP (MBP21.5) in the aged mice, which is known to be closely related to the demyelination status [22, 32], was also improved in the Chinpi-treated mice (Figure 5).

3.3. Effect of Chinpi Compounds on OPC Proliferation In Vitro. The effect of two specific compounds of Chinpi (hesperidin plus narirutin) was investigated at the cellular levels using in vitro OPC cultures (Figure 6(a)). Purified OPCs were incubated with Chinpi components $(10 \mu \mathrm{g} / \mathrm{mL}$ hesperidin plus $10 \mu \mathrm{g} / \mathrm{mL}$ narirutin) and cell proliferation was assessed after $48 \mathrm{~h}$ by double immunostaining for BrdU incorporation (a proliferation marker) and Ddx54 antibody. As shown in Figure 6(b), the addition of Chinpi ingredients increased the ratio of BrdU-positive cells, and the increase was due to the proliferation of Ddx $54^{+}$OPCs, not Olig2 ${ }^{+}$OPCs or $\mathrm{NG}^{+}$ OPCs.

\section{Discussion}

Aging is a primary factor that impairs remyelination, as observed in neurodegenerative diseases such as Alzheimer's disease in which patients develop progressive cognitive decline. Cells of the oligodendrocyte lineage, specifically OPCs, may be targeted as source of remyelination because they are present in the adult brain parenchyma of not only mice but also humans $[22,32]$. Neural stem cells in the adult subventricular zone of the lateral ventricles may also represent an alternative source of OPCs for remyelination [33]. Therefore it is possible that the promotion of proliferation, recruitment, and differentiation of OPCs in the lesion site is a promising therapeutic strategy to repair myelin deficits. Only a few factors are known to induce proliferation, differentiation, and motility in OPCs $[34,35]$.

The present study demonstrated that two months of treatment with a water-soluble extract of Chinpi, administered via the drinking water, reversed aging-induced demyelination in mice (Figure 1). Furthermore, Chinpi was associated with an increase in Ddx54-expressing cells in the ventricular facing side of the lateral ventricle and corpus callosum (Figures 3 and 4). Ddx54 is considered to be a specific marker of the oligodendrocyte linages from the embryonic stage to adulthood [20], and it is indispensable for both the OPC maturation (i.e., production of MBP21.5) and translocation from the ventricular zone to the corpus callosum [21]. Furthermore, close relationships between expression levels of Ddx54 and MBP21.5 and myelination status have been demonstrated in different demyelination models (cuprizone, aging and FcR $\gamma /$ Fyn double knockout) $[21,23,36]$. This suggests that the Ddx54-expressing cells observed in the present study may represent newly generated OPCs, which are destined to be myelinating oligodendrocytes for efficient remyelination (Figure 7).

Of note, Chinpi did not attenuate the loss of Ddx54expressing OPCs during the two-month treatment period until the mice were 30 months old but actively increased the number of Ddx54-expressing cells. This was demonstrated by the observation that Ddx54 immunoreactivity was negligible in both the 28-month-old control mice and the 30-monthold control mice (Supplementary Fig. S1). Although NSCs are found in the subventricular zone of the aged brain, their proliferative and neurogenic capacity are diminished with age [37]. However, the reappearance of Ddx54-expressing cells in the subventricular zone following Chinpi treatment suggests that a proportion of NSCs may be capable of reactivation to produce new oligodendrocyte lineages, presumably leading 


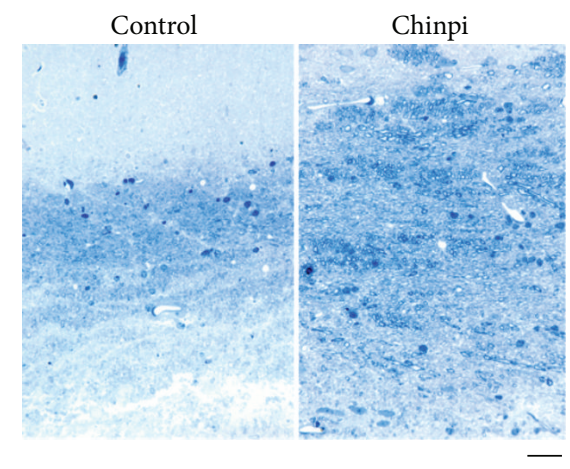

(a)

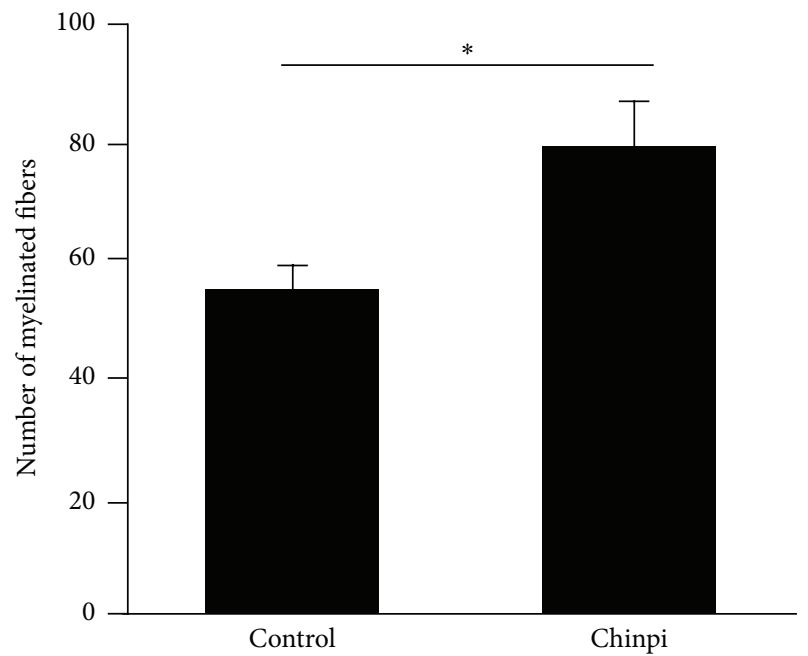

(c)

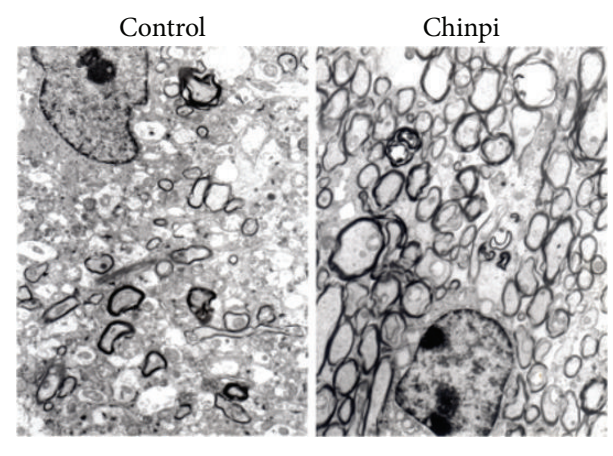

(b)
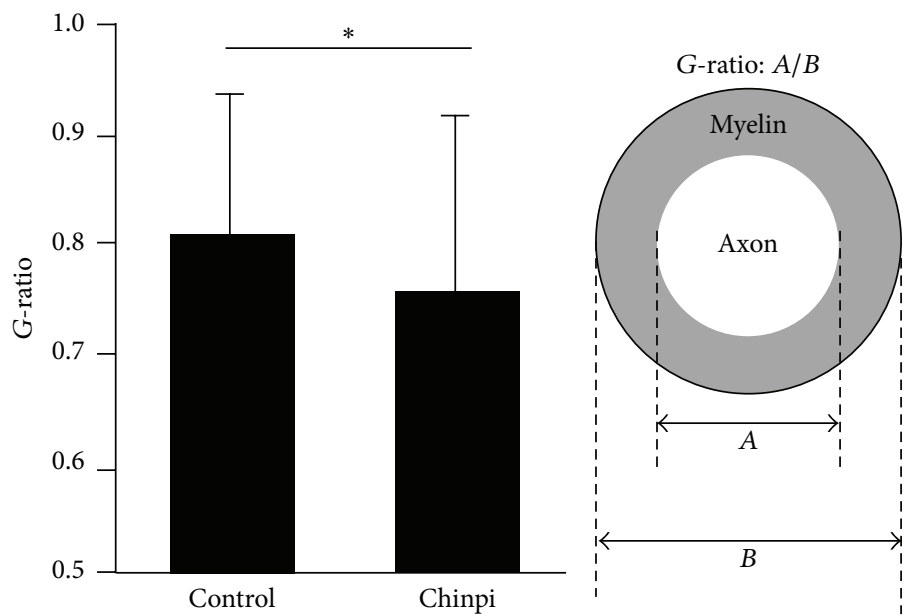

(d)

FIGURE 2: (a) Toluidine blue staining of cross sections of the corpus callosum. Myelin staining was increased in Chinpi-treated mice. Scale bar $=85 \mu \mathrm{m}$. (b) Electron micrographs of cross section of the corpus callosum show aging-induced demyelination in control mice. The myelination status was improved after two months of Chinpi treatment. Scale bar $=2 \mu \mathrm{m}$. (c) The density of myelinated fibers per $300 \mu \mathrm{m}^{2}$. Treatment with Chinpi led to a significant increase in the myelinated nerve fiber density. The number of myelinated fibers was counted using five electron micrograph images from each mouse. Data represent mean $\pm \operatorname{SEM}(n=3):{ }^{*} P<0.05$. (d) The $G$-ratio, an indicator of demyelination, which is defined as the ratio of the axon diameter to that of the axon diameter plus the surrounding myelin sheathe (see right panel for formula) was calculated. The increased $G$-ratio in the elderly mice was improved by Chinpi treatment. The number of measured axons was 90 , using eight electron micrographs from three mice. Data represent mean \pm SEM. ${ }^{*} P<0.05$ versus control. The analyses were performed using 30-month-old mice.

to remyelination. In accordance, in vitro experiments with hesperidin and narirutin (two of the active constituents of Chinpi) revealed a significant increase in the ratio of BrdU incorporation, indicative of cell proliferation into $\mathrm{Ddx} 54^{+}$ cells (Figure 4 ). The present findings strongly suggest that reactivation of "resting" NSC in the aged brain, as exemplified by Chinpi, may be a promising strategic option for the treatment of aging-induced demyelination.

Ddx54 is a specific marker for oligodendrocytes lineages, including OPCs; however, Ddx54 expression is not expressed in all Olig2 ${ }^{+}$and/or NG2 ${ }^{+}$OPCs (Supplementary Fig. S2). Similarly, only a subset of $\mathrm{O}^{+}$immature, $\mathrm{O}^{+}$immature, and/or $\mathrm{MBP}^{+}$mature oligodendrocytes express Ddx54 protein [20]. Many OPCs are generated in the brain; however, only a small proportion of these differentiate and attain the ability myelinate axons. The vital molecules necessary for maturation of OPCs into myelinating oligodendrocytes are largely unknown, but Ddx54 may be a candidate molecule (Figure 7). Ddx54 binds to both the mRNA and protein of MBP isoforms: binding to mouse MBP mRNA was previously demonstrated [20] and binding to mouse and rat MBP proteins is shown in supplementary Fig. S3 and the previous report [20], respectively. Ddx54 is a DEAD box RNA-helicase that is implicated in the key steps of RNA-related processes, including transcription, pre-mRNA processing, ribosome biogenesis, RNA export, translational initiation, and RNA degradation $[16,38]$. Our previous studies demonstrated that Ddx54 knockdown inhibits the intrusion of OPCs from the ventricular zone to the corpus callosum, resulting in the failure of axonal myelination. We have also reported that oligodendrocytes cultured under oxygen-glucose deprivation lose the ability to interact with neuron and also display 


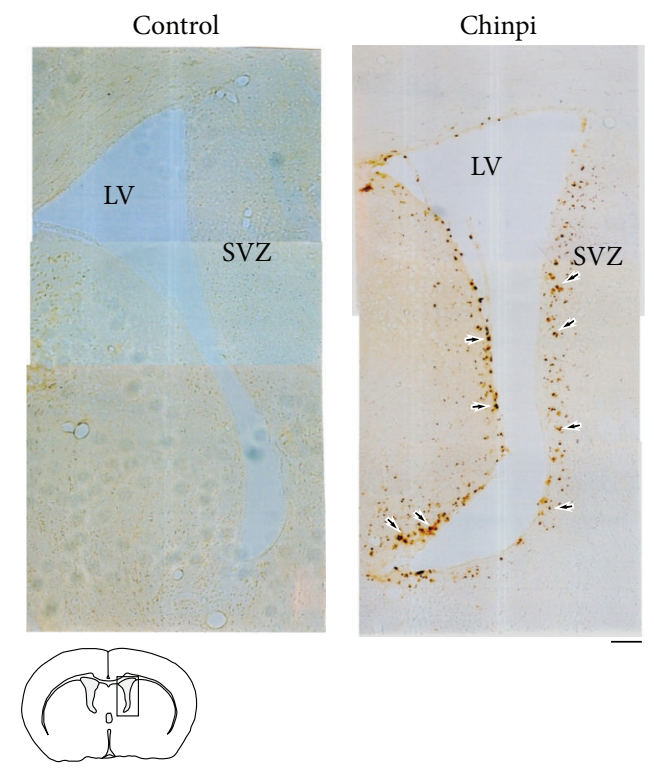

FIGURE 3: Immunohistochemistry of sections of the ventricular (VZ) and subventricular zone (SVZ) of the lateral ventricles (LV) with anti-Ddx54 antibody. Ddx54 immunoreactivity was negligible in the control specimens. Strong Ddx54 immunoreactivity but with an uneven distribution was observed in Chinpi-treated brains. Scale bar $=28 \mu \mathrm{m}$. The analyses were performed using 30-month-old mice.

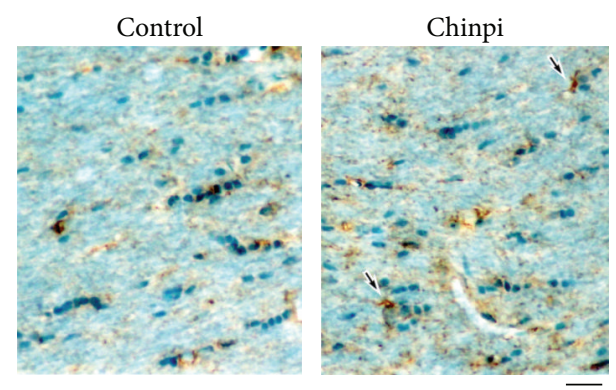

FIGURE 4: Immunohistochemistry of corpus callosum sections stained with anti-Ddx54 antibody. In Chinpi-treated mice, Ddx54expressing cells with the morphology of immature/mature oligodendrocytes (arrows) were observed, while in the control mice such cells were scarcely detected. Scale bar $=112 \mu \mathrm{m}$. This experiment was performed using 30-month-old mice.

reduced expression of Ddx54 and MBP21.5 [36]. These findings address the possibility that Ddx54 may play a critical role in MBP mRNA transport, "on-site" synthesis of MBP protein in the myelin sheath, and therefore axonal contact. To fully elucidate the biological roles in Ddx54, extensive analyses using transgenic and/or knockout Ddx54 models are necessary. Such studies are currently underway.

\section{Conclusion}

The present findings suggest a novel therapeutic strategy for the aging-induced decline in the efficiency of remyelination. Chinpi and Chinpi-containing Japanese traditional (Kampo)

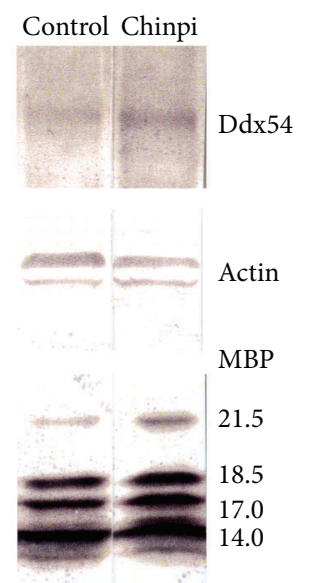

FIGURE 5: Western blotting of brain homogenates from control and Chinpi-treated aged mice (at 30 months old). The signal intensity of both Ddx 54 protein and $21.5-\mathrm{kDa}$ isoform of MBP was higher in Chinpi-treated mice compared with control mice.

medicines are widely used in clinics in Japan. In particular, Kampo medicines of pharmaceutical grade are manufactured under strict scientific control of active ingredients and impurities and integrated in national insurance systems resulting in the widespread use of Kampo medicines in almost all national, public, and university hospitals as well as private clinics. These medicines may provide a readily available means for the treatment of various demyelinating diseases. Furthermore, better elucidation of the mechanisms by which Chinpi promotes remyelination will greatly contribute to the scientific understanding of the biological processes underlying myelination and remyelination.

\section{Abbreviations}

BrdU: 5-Bromo-2'-deoxyuridine

CNS: Central nervous system

LV: $\quad$ Lateral ventricles

MBPs: Myelin basic proteins

NSCs: Neural stem cells

OPCs: Oligodendrocyte progenitor cells

SVZ: Subventricular zone

V-SVZ: Ventricular-subventricular zone

VZ: Ventricular zone.

\section{Highlights}

The highlights are as follows:

(i) Chinpi (citrus peel) reverses aging-induced demyelination.

(ii) Chinpi increased Ddx54-expressing OPCs in vivo and in vitro.

(iii) $\mathrm{Ddx} 54^{+} 21.5 \mathrm{kDa} \mathrm{MBP}^{+}$OPC may be critical for myelination/remyelination. 

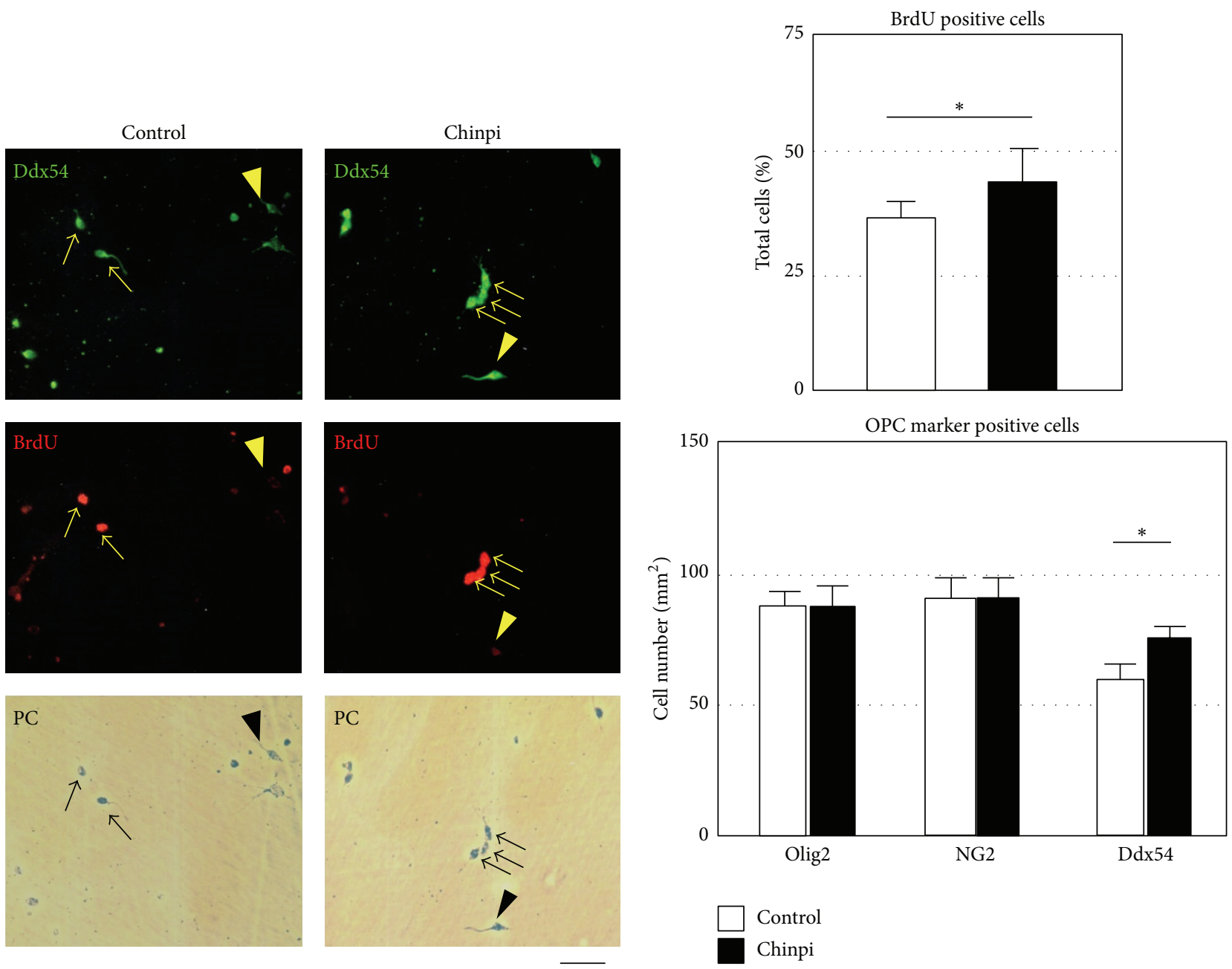

(a)

(b)

Figure 6: (a) Immunofluorescence staining of purified OPC cultures with antibodies against Ddx 54 and BrdU. At 48 hr after addition of $20 \mu \mathrm{M}$ BrdU, cells cultured with or without hesperidin plus narirutin $(10 \mu \mathrm{g} / \mathrm{mL}$ each) were stained with anti-Ddx 54 (top row, green fluorescence) and anti-BrdU antibodies (middle row, red fluorescence). Ddx54-positive cells (arrows in top row), BrdU-positive cells (arrows in middle row), and phase contrast images (bottom row) were shown. Arrowhead indicates BrdU-negative cells. Scale bar $=20 \mu \mathrm{m}$. (b) Quantitation of proliferating OPCs by double immunofluorescence staining of purified OPC cultures with antibodies for OPC markers and anti-Ddx54 antibody. Top row: percentage of BrdU ${ }^{+}$cells of total cells. Bottom row: the number of the cells coexpressing BrdU and various OPC markers. Selective proliferation of Ddx $54^{+}$cells, but not in Olig2 ${ }^{+}$or $\mathrm{NG}^{+}$cells, was induced by Chinpi ingredients resulting in an increase in the total number of cultured cells. Data are represented as mean \pm SEM. ${ }^{*} P<0.05$ versus control.

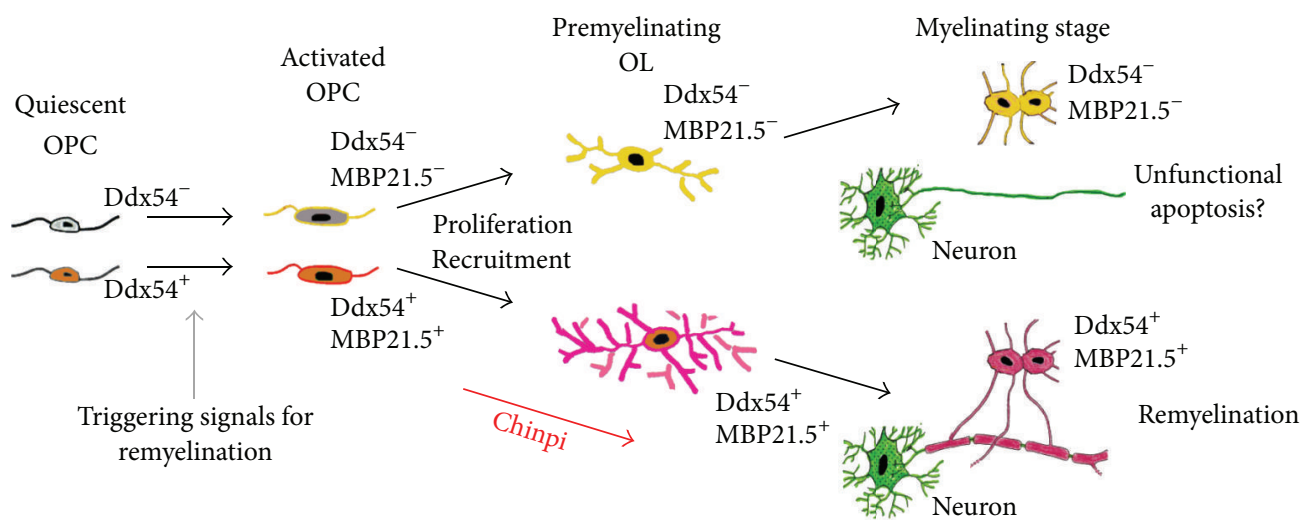

FIGURE 7: A hypothesis of the fate of oligodendrocyte linages in remyelination. Demyelination triggers the proliferation and recruitment of OPC. Ddx54 ${ }^{+}$OPC differentiate into MBP21.5-expressing oligodendrocytes (OL) that obtain myelinating capacity, while Ddx54- OPC differentiate into MBP21.5 $\mathrm{OL}$ that cannot myelinate axons. 


\section{Conflict of Interests}

Masahiro Yamamoto is an employee of Tsumura \& Co. which is a pharmaceutical company of Kampo medicines. Hiroaki Asou received the research grant from Tsumura \& Co. Other authors declare that there is no conflict of interests regarding the publication of this paper.

\section{Authors' Contribution}

Hideaki Tokunaga and Chika Seiwa have contributed equally to this work.

\section{References}

[1] S. P. J. Fancy, C. Zhao, and R. J. M. Franklin, "Increased expression of Nkx2.2 and Olig2 identifies reactive oligodendrocyte progenitor cells responding to demyelination in the adult CNS," Molecular and Cellular Neuroscience, vol. 27, no. 3, pp. 247-254, 2004.

[2] L. E. Rivers, K. M. Young, M. Rizzi et al., "PDGFRA/NG2 glia generate myelinating oligodendrocytes and piriform projection neurons in adult mice," Nature Neuroscience, vol. 11, no. 12, pp. 1392-1401, 2008.

[3] J. M. Gensert and J. E. Goldman, "Endogenous progenitors remyelinate demyelinated axons in the adult CNS," Neuron, vol. 19, no. 1, pp. 197-203, 1997.

[4] R. J. M. Franklin, J. M. Gilson, and W. F. Blakemore, "Local recruitment of remyelinating cells in the repair of demyelination in the central nervous system," Journal of Neuroscience Research, vol. 50, no. 2, pp. 337-344, 1997.

[5] B. Menn, J. M. Garcia-Verdugo, C. Yaschine, O. Gonzalez-Perez, D. Rowitch, and A. Alvarez-Buylla, "Origin of oligodendrocytes in the subventricular zone of the adult brain," The Journal of Neuroscience, vol. 26, no. 30, pp. 7907-7918, 2006.

[6] B. Nait-Oumesmar, L. Decker, F. Lachapelle, V. Avellana-Adalid, C. Bachelin, and A. Baron-Van Evercooren, "Progenitor cells of the adult mouse subventricular zone proliferate, migrate and differentiate into oligodendrocytes after demyelination," European Journal of Neuroscience, vol. 11, no. 12, pp. 4357-4366, 1999.

[7] N. Picard-Riera, L. Decker, C. Delarasse et al., "Experimental autoimmune encephalomyelitis mobilizes neural progenitors from the subventricular zone to undergo oligodendrogenesis in adult mice," Proceedings of the National Academy of Sciences of the United States of America, vol. 99, no. 20, pp. 13211-13216, 2002.

[8] M. Cayre, J. Malaterre, S. Scotto-Lomassese, C. Strambi, and A. Strambi, "The common properties of neurogenesis in the adult brain: from invertebrates to vertebrates," Comparative Biochemistry and Physiology Part B: Biochemistry and Molecular Biology, vol. 132, no. 1, pp. 1-15, 2002.

[9] J. A. Ortega, N. V. Radonjić, and N. Zecevic, "Sonic hedgehog promotes generation and maintenance of human forebrain Olig2 progenitors," Frontiers in Cellular Neuroscience, vol. 7, article 254, 2013.

[10] J. Gilson and W. F. Blakemore, "Failure of remyelination in areas of demyelination produced in the spinal cord of old rats," Neuropathology and Applied Neurobiology, vol. 19, no. 2, pp. 173181, 1993.
[11] S. A. Shields, J. M. Gilson, W. F. Blakemore, and R. J. M. Franklin, "Remyelination occurs as extensively but more slowly in old rats compared to young rats following gliotoxin-induced CNS demyelination," Glia, vol. 28, no. 1, pp. 77-83, 1999.

[12] B. A. Shook, D. H. Manz, J. J. Peters, S. Kang, and J. C. Conover, "Spatiotemporal changes to the subventricular zone stem cell pool through aging," Journal of Neuroscience, vol. 32, no. 20, pp. 6947-6956, 2012.

[13] S. Mi, R. H. Miller, W. Tang et al., "Promotion of central nervous system remyelination by induced differentiation of oligodendrocyte precursor cells," Annals of Neurology, vol. 65, no. 3, pp. 304-315, 2009.

[14] A. Nishiyama, M. Komitova, R. Suzuki, and X. Zhu, "Polydendrocytes (NG2 cells): multifunctional cells with lineage plasticity," Nature Reviews Neuroscience, vol. 10, no. 1, pp. 9-22, 2009.

[15] R. J. M. Franklin, "Why does remyelination fail in multiple sclerosis?" Nature Reviews Neuroscience, vol. 3, no. 9, pp. 705$714,2002$.

[16] P. Linder, "Dead-box proteins: a family affair-active and passive players in RNP-remodeling," Nucleic Acids Research, vol. 34, no. 15, pp. 4168-4180, 2006.

[17] G. L. Hinks and R. J. M. Franklin, "Delayed changes in growth factor gene expression during slow remyelination in the CNS of aged rats," Molecular and Cellular Neuroscience, vol. 16, no. 5, pp. 542-556, 2000.

[18] G. Wolswijk and M. Noble, "Cooperation between PDGF and FGF converts slowly dividing O-2Aadult progenitor cells to rapidly dividing cells with characteristics of O-2Aperinatal progenitor cells," Journal of Cell Biology, vol. 118, no. 4, pp. 889900, 1992.

[19] G. Wolswijk, P. N. Riddle, and M. Noble, "Platelet-derived growth factor is mitogenic for O-2Aadult progenitor cells," Glia, vol. 4, no. 5, pp. 495-503, 1991.

[20] T. Ueki, Y. Tsuruo, Y. Yamamoto et al., "A new monoclonal antibody, 4F2, specific for the oligodendroglial cell lineage, recognizes ATP-dependent RNA helicase Ddx54: possible association with myelin basic protein," Journal of Neuroscience Research, vol. 90, no. 1, pp. 48-59, 2012.

[21] R. Zhan, M. Yamamoto, T. Ueki et al., "A DEAD-box RNA helicase Ddx54 protein in oligodendrocytes is indispensable for myelination in the central nervous system," Journal of Neuroscience Research, vol. 91, no. 3, pp. 335-348, 2013.

[22] C. Seiwa, M. Yamamoto, K. Tanaka et al., "Restoration of FcR $\gamma /$ Fyn signaling repairs central nervous system demyelination," Journal of Neuroscience Research, vol. 85, no. 5, pp. 954966, 2007.

[23] M. Uruse, M. Yamamoto, M. Sugawa et al., "Phase separation of myelin sheath in Triton X-114 solution: predominant localization of the $21.5-\mathrm{kDa}$ isoform of myelin basic protein in the lipid raft-associated domain," The Journal of Biochemistry, vol. 155, no. 4, pp. 265-271, 2014.

[24] H. Asou, N. Sato, C. Seiwa et al., "Administration of chinpi, a component of the herbal medicine ninjin-youei-to, reverses age-induced demyelination," Evidence-Based Complementary and Alternative Medicine, vol. 2011, Article ID 617438, 9 pages, 2011.

[25] N. Yoshioka, S.-I. Hisanaga, and H. Kawano, "Suppression of fibrotic scar formation promotes axonal regeneration without disturbing blood-brain barrier repair and withdrawal of leukocytes after traumatic brain Injury," Journal of Comparative Neurology, vol. 518, no. 18, pp. 3867-3881, 2010. 
[26] N. Yoshioka, J. Kimura-Kuroda, T. Saito, K. Kawamura, S.-I. Hisanaga, and H. Kawano, "Small molecule inhibitor of type I transforming growth factor- $\beta$ receptor kinase ameliorates the inhibitory milieu in injured brain and promotes regeneration of nigrostriatal dopaminergic axons," Journal of Neuroscience Research, vol. 89, no. 3, pp. 381-393, 2011.

[27] K. Akiyama, S. Ichinose, A. Omori, Y. Sakurai, and H. Asou, "Study of expression of myelin basic proteins (MBPs) in developing rat brain using a novel antibody reacting with four major isoforms of MBP," Journal of Neuroscience Research, vol. 68, no. 1, pp. 19-28, 2002.

[28] C. Seiwa, K. Kojima-Aikawa, I. Matsumoto, and H. Asou, “CNS myelinogenesis in vitro: myelin basic protein deficient shiverer oligodendrocytes," Journal of Neuroscience Research, vol. 69, no. 3, pp. 305-317, 2002.

[29] I. Sugiyama, K. Tanaka, M. Akita, K. Yoshida, T. Kawase, and H. Asou, "Ultrastructural analysis of the paranodal junction of myelinated fibers in 31-month-old-rats," Journal of Neuroscience Research, vol. 70, no. 3, pp. 309-317, 2002.

[30] K. L. Baracskay, G. J. Kidd, R. H. Miller, and B. D. Trapp, "NG2-positive cells generate A2B5-positive oligodendrocyte precursor cells," Glia, vol. 55, no. 10, pp. 1001-1010, 2007.

[31] K. Yoshimura, Y. Sakurai, D. Nishimura et al., "Monoclonal antibody 14F7, which recognizes a stage-specific immature oligodendrocyte surface molecule, inhibits oligodendrocyte differentiation mediated in co-culture with astrocytes," Journal of Neuroscience Research, vol. 54, no. 1, pp. 79-96, 1998.

[32] G. Harauz and J. M. Boggs, "Myelin management by the 18.5$\mathrm{kDa}$ and 21.5-kDa classic myelin basic protein isoforms," Journal of Neurochemistry, vol. 125, no. 3, pp. 334-361, 2013.

[33] C.-W. Hung, Y.-J. Liou, S.-W. Lu et al., "Stem cell-based neuroprotective and neurorestorative strategies," International Journal of Molecular Sciences, vol. 11, no. 5, pp. 2039-2055, 2010.

[34] F. Jiang, T. J. Frederick, and T. L. Wood, "IGF-I synergizes with FGF-2 to stimulate oligodendrocyte progenitor entry into the cell cycle," Developmental Biology, vol. 232, no. 2, pp. 414-423, 2001.

[35] P. Ye, J. Carson, and A. J. D’Ercole, "In vivo actions of insulinlike growth factor-I (IGF-I) on brain myelination: studies of IGF-I and IGF binding protein-1 (IGFBP-1) transgenic mice," The Journal of Neuroscience, vol. 15, no. 11, pp. 7344-7356, 1995.

[36] M. Ichinose, Y. Kamei, T. Iriyama et al., "Hypothermia attenuates apoptosis and protects contact between myelin basic protein-expressing oligodendroglial-lineage cells and neurons against hypoxia-Ischemia," Journal of Neuroscience Research, vol. 92, no. 10, pp. 1270-1285, 2014.

[37] V. Capilla-Gonzalez, V. Herranz-Pérez, and J. M. GarcíaVerdugo, "The aged brain: genesis and fate of residual progenitor cells in the subventricular zone," Frontiers in Cellular Neuroscience, vol. 9, article 365, 2015.

[38] O. Cordin, J. Banroques, N. K. Tanner, and P. Linder, "The DEAD-box protein family of RNA helicases," Gene, vol. 367, no. 1-2, pp. 17-37, 2006. 


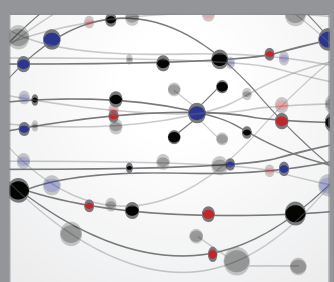

The Scientific World Journal
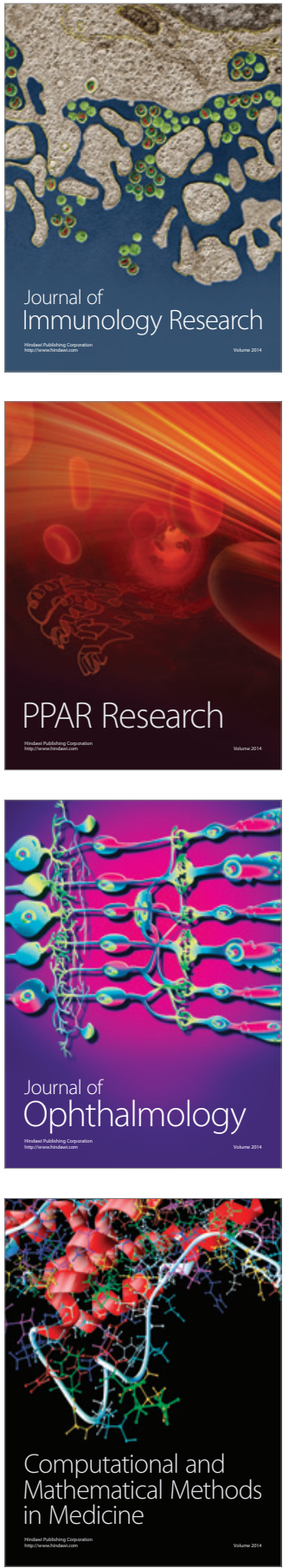

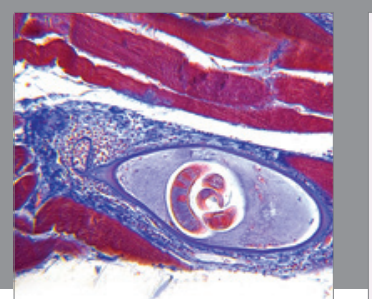

Gastroenterology Research and Practice

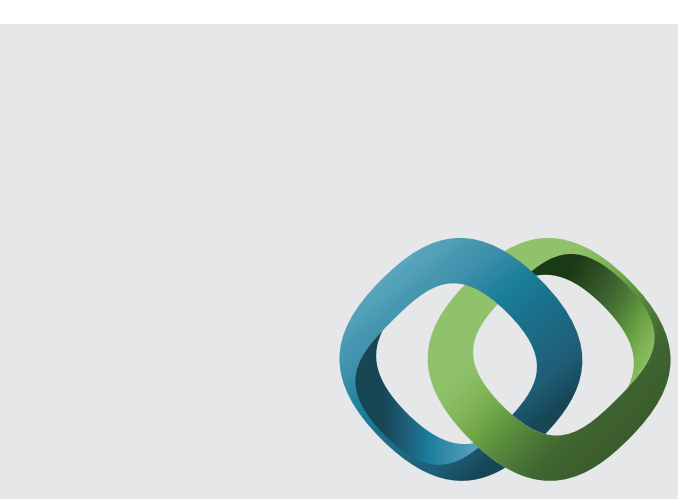

\section{Hindawi}

Submit your manuscripts at

http://www.hindawi.com
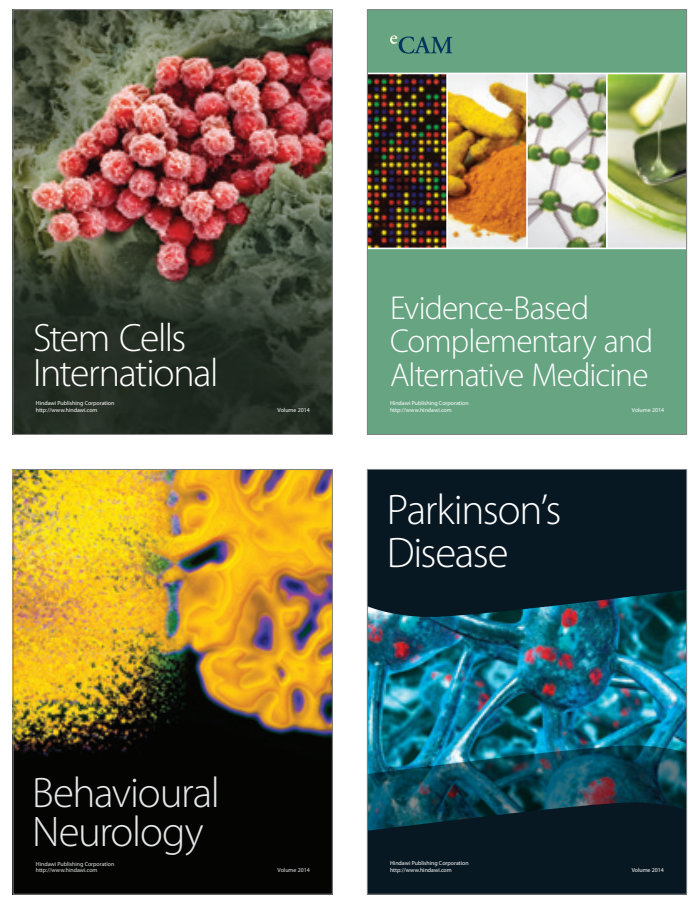
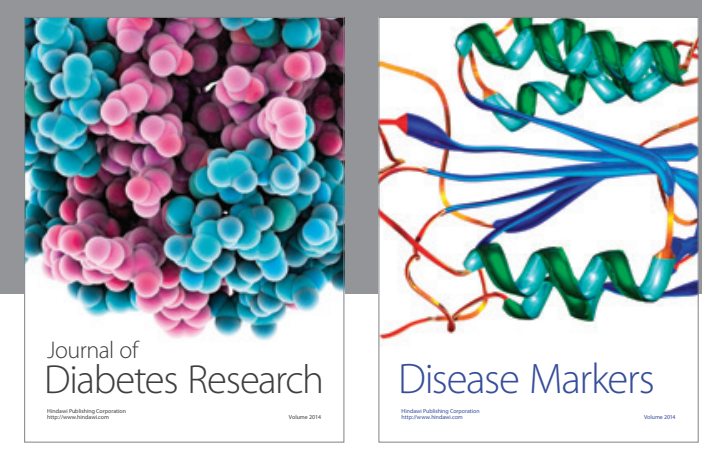

Disease Markers
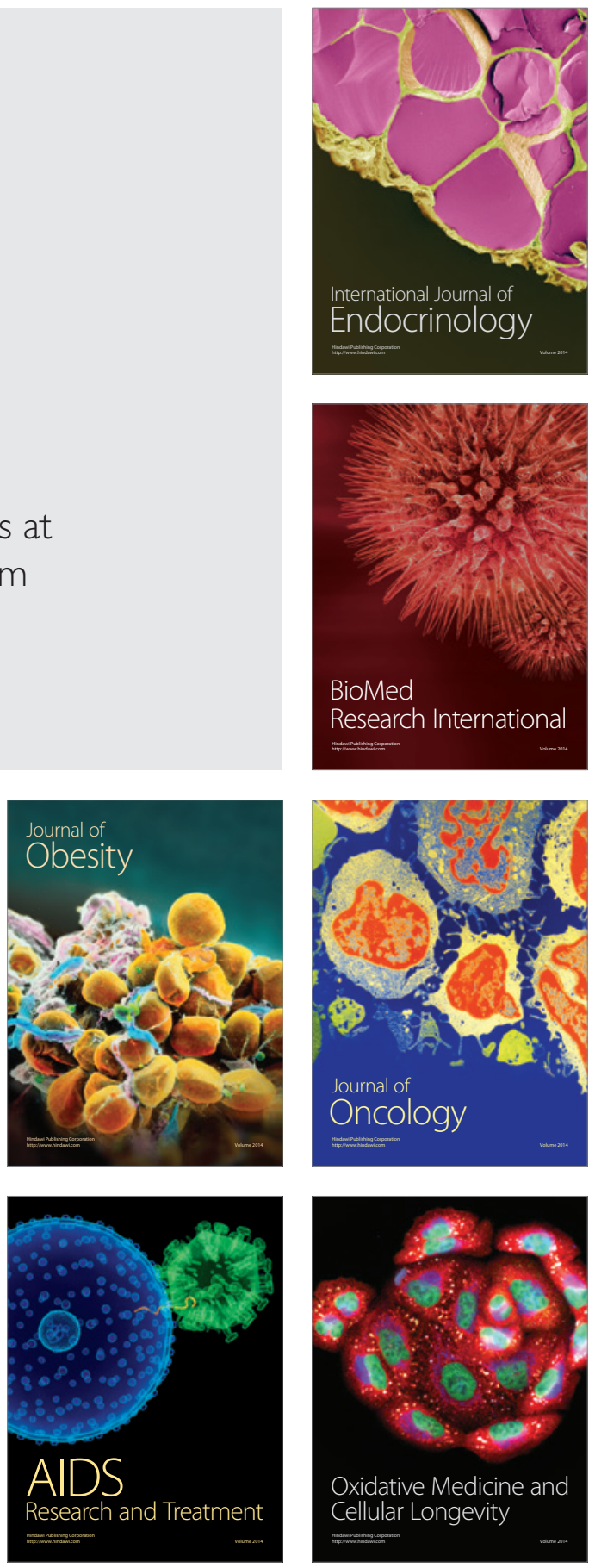